\title{
AIDS-Related Behavioral Characteristics of Men Who Have Sex with Men in Chongqing, China
}

\author{
Yanran Ou ${ }^{1-3}$, Hong Pan ${ }^{1-3}$, Jiaxiu Liu ${ }^{1-3}$, Bing Lin ${ }^{1-3}$, Guiqian Shi ${ }^{1-3}$ and Xiaoni Zhong ${ }^{1-3, *}$ \\ ${ }^{1}$ School of Public Health and Management, Chongqing Medical University, Chongqing, China \\ ${ }^{2}$ Research Center for Medicine and Social Development, Chongqing Medical University, Chongqing, China \\ ${ }^{3}$ Innovation Center for Social Risk Governance in Health, Chongqing Medical University, Chongqing, China
}

*Corresponding author: Xiaoni Zhong, School of Public Health and Management, Chongqing Medical University, Chongqing, China, Tel: +861330-836-8059; E-mail: zhongxiaoni@cqmu.edu.cn

Received: 28 May, 2020 | Accepted: 19 Jun, 2020 | Published: 26 Jun, 2020

Citation: Ou Y, Pan H, Liu J, Lin B, Shi G, et al. (2020) AIDS-Related Behavioral Characteristics of Men Who Have Sex with Men in Chongqing, China. J Epidemiol Public Health Rev 5(2): dx.doi.org/10.16966/2471-8211.193

Copyright: (c) 2020 Ou Y, et al. This is an open-access article distributed under the terms of the Creative Commons Attribution License, which permits unrestricted use, distribution, and reproduction in any medium, provided the original author and source are credited.

\begin{abstract}
Background: Men who have sex with men (MSM) are a high-risk group of HIV infection, and the prevalence of HIV in MSM of Chongqing, China is relatively high. For the effective prevention of HIV, this study aimed to understand the AIDS-related demographic and behavioral characteristics of MSM population in Chongqing, and provide references for the targeted prevention and control of AIDS.

Methods: In this study, One hundred and fifteen MSM aged 18 to 65 were recruited voluntarily through online recruitment, "snowball method" (initial subjects were utilized to recruit peers), and peer introduction. Social demographic data including age, marital status, education level, employment, and sexual behavior characteristics including the number of sexual partners, condom use, and other factors related to HIV infection were collected.

Results: Most of the MSM in the study were $18-33$ years old (45.2\%) and highly educated $(50.4 \%$ were junior college or above), $65.2 \%$ were unmarried, $80 \%$ were employed, and $40.0 \%$ had a highly monthly income (more than $5000 \mathrm{RMB}$ ). In addition, the majority had multiple sexual partners and unprotected insertive sex behavior. Besides, bisexual behavior was common among MSM population. Among those MSM who reported regular female partners, nearly half of them did not always use condoms when having sex with wives or girlfriends (45.9\%).

Conclusions: There are multiple sexual partners, unprotected inserted sex, bisexuality and other behaviors in the MSM population, which are risk factors for HIV infection. This study recommends taking appropriate prevention and control measures against the above findings. Strengthening AIDS risk knowledge popularization and reducing sexual partners should be recommended to this group, so as to reduce the opportunity of contacting various sexual behaviors and potential partners with HIV positive. For reducing the risk of HIV infection in MSM and male partners when they have insertive sex, they should be advised to use condoms correctly and throughout. In order to reduce the possibility of HIV transmission to HIV negative partners, pre-marital and pregnancy tests including HIV testing should be provided to married couples.
\end{abstract}

Keywords: MSM; AIDS-related; Behavioral characteristics; China

\section{Introduction}

After the first case of acquired immunodeficiency syndrome (AIDS) was reported in the United States [1], the prevalence of HIV was frequently reported worldwide and the reporting of AIDS increases year by year [2]. According to The United Nations AIDS Program (UNAIDS), by 2018, the number of people living with AIDS is about 37.9 million (32.7-44 million) [3,4]. As one of the most serious infectious diseases in the world, AIDS has threatened the health of people, and the latest date (2018) reveals 0.86 million people in China already know that they are infected with HIV [5].

UNAIDS believes that gay men and other men who have sex with men (MSM), sex workers, transgender people, injecting drug users, and prisoners are particularly susceptible to HIV [6], which were considered to be a key population of HIV infection $[4,6]$. In 2018, more than half of the new HIV infections worldwide were key population and their sexual partners, and about $17 \%$ of the newly infected were gay men and other men who have sex with men [4]. Besides, in the Asia-Pacific region, more than three-quarters of new HIV infections were key populations and their sexual partners, of whom about $30 \%$ of newly infected people were MSM [4].

In China, MSM is a high-risk group for HIV infection [6-8]. National AIDS sentinel surveillance data showed that the HIV infection rate of MSM in China (6.9\%) was higher than that of sex workers $(0.2 \%)$ and injecting drug users (5.9\%) in 2018 [5]. In recent years, various provinces, cities, and autonomous regions in China have discovered the prevalence of HIV in MSM and the HIV infection rate have increased significantly $[6,9]$, especially in the southwest region $[6,9,10]$. According to reports, most of the HIV-infected MSM 
in China are the young and middle-aged population aged 20-39 [7], and have a low level of education [10-12]. Moreover, most of the recent HIV-infected MSM are young people $[4,11,13]$ and the elderly [11] in China. Meanwhile the risky behaviors of unprotected sexual intercourse (rarely using condoms) are common in MSM when having sex with sexual partners $[6,10,12,14]$, and some MSM have multiple sexual partners $[6,12]$. In addition, MSM usually have multiple risk behaviors such as sex trade [6,14], and injecting drugs [6].

Previously studies have found that the main mode of transmission of AIDS in China is sexual transmission [11,13], and the infection rate through homosexual transmission has increased $[7,11]$. Besides, MSM is susceptible to HIV infections from both genders due to bisexuality or marriage with women under social and family pressures [10], and then HIV is transmitted by inserting sexual behaviors (via vagina or anus sex, especially anal sex) [15], thus MSM are becoming a bridge for HIV transmission to others [9-11,14].

At present, MSM have become a concern group for AIDS prevention [12]. In order to reduce the risk of AIDS transmission among MSM populations and prevent the further spread of HIV among HIVnegative MSM populations, this study focused on MSM population, and investigated information such as age, education level, marital status, condom use, sexual partner status and so on. By analyzing the demographic and behavioral characteristics of MSM, this study aimed to provide a theoretical basis for the preventive measures targeted for this group.

\section{Materials and Methods}

\section{Participants and procedure}

MSM aged 18 to 65 years and willing to participate in the survey were selected as the subjects of this study.

\section{Recruitment strategy}

From December 2019 to February 2020, participants were recruited by online recruitment, core members "snowball", peer introduction or recruitment through their organizations in Chongqing, China, in order to recruit as many MSM as possible.

Recruitment through non-governmental organization (NGO) of the MSM population: Contacting key personnel of the NGO organization, publicizing and introducing the research to MSM including the research content, potential benefits and risks, obtaining their support, and then mobilizing and recruiting other people of the NGO through them.

Training MSM peer educators to promote and recruit in the entertainment or public places frequented by MSM: Places include bars, bathrooms, and parks where posters and leaflets can be distributed to publicize after obtaining the consent and support of the venue operators.

Recruitment was carried out through HIV Voluntary Counseling \& Testing (VCT) clinic. In each VCT clinic, MSM who come to HIV counseling and testing will be explained and publicized. For those who are willing to participate in the study, their contact information will be left, and then they will be introduced to the research site for screening and evaluation.

Snowball method is a method that relies on initial subjects (seeds) to recruit others (their sexual partners or peers) from their social networks [16]. The detailed method is as follows: select several MSM with the required characteristics as the initial subjects firstly then they are relied on to provide qualified survey respondents, the third group of subjects are provided by the latter, and the next steps by analogy.

Online recruitment: Recruitment was conducted by publishing information about this study on MSM website or QQ group.

Existing MSM queue: Recruit from the MSM population in the $12^{\text {th }}$ Five Year Plan AIDS program or other programs in Chongqing, China.

\section{HIV-1 serological testing}

The venous blood of volunteers who participated in the study was collected and sent to the First Affiliated Hospital of Chongqing Medical University for HIV-1 serological testing by Enzyme-Linked Immunosorbent Assay (ELISA).

\section{Data extraction and statistical analysis}

Establish database: EpiData3.0 software was used to establish the corresponding entry procedure, and set the logical review limit conditions during entry, carry out the trial operation on the database, and then establish a database system dedicated for this study. Double entry method was used to input data, and the verification function in EpiData3.0 software was used to check the data, so as to ensure the accuracy of the entered data.

Data extraction: Age, education level, employment, marital status, monthly income and other demographic data from the database were collected, as well as behaviors such as the number of sexual partners, the number of insertions (anal sex and oral sex), and condom use frequency.

Statistical analysis: SAS9.4 statistical analysis software was used for analysis. Composition ratio was used for description, and the mode was used to fill in missing data.

\section{Quality control and ethics}

This study was approved by the Ethics Committee of Chongqing Medical University (approval number: 2019001), and the participants all signed the informed consent before this trial. During the investigation, the subjects were completely voluntary and did not suffer any harm due to their participation in the investigation. And the personal information of the subjects had been kept strictly confidential to protect their privacy. For those who were negative for HIV antibody testing, we had timely informed the accurate test results, and provided corresponding AIDS publicity and consulting services; for those who were HIV antibody positive, we quickly contacted the subjects themselves in accordance with the procedure, and then went to the local Centers for Disease Control and Prevention (CDC) for follow-up confirmation test and corresponding services, so that the infected subjects could receive professional consultation and guidance provided by the medical staffs, which was beneficial to infected persons or patients to control opportunistic infections, prolong life, and improve quality of life. It could also avoid the spread of the virus in the family and protect their families.

\section{Results}

\section{Social demographic characteristics}

Among the 115 MSM subjects in this study, unmarried people and young people aged 18-33 accounted for a large proportion, accounting for $65.2 \%$ and $45.2 \%$ respectively. The majority of MSM had a junior college degree or above, accounting for $50.4 \%(n=58)$, of which the bachelor degree or above accounted for about one third of the subjects. Most of them had a steady job, accounting for $80.0 \%(n=92)$. In addition, the proportion of people with a higher monthly income level (more than $5000 \mathrm{RMB})$ was $40.0 \%(\mathrm{n}=46)$ (Table 1$)$. 
Table 1: Social demographic characteristics of MSM in Chongqing.

\begin{tabular}{|c|c|c|}
\hline Characteristics & $\mathbf{n}$ & $\%$ \\
\hline \multicolumn{3}{|l|}{ Age } \\
\hline$<18$ & 0 & 0 \\
\hline $18-33$ & 52 & 45.2 \\
\hline $34-44$ & 36 & 31.3 \\
\hline $45-59$ & 20 & 17.4 \\
\hline$\geq 60$ & 7 & 6.1 \\
\hline \multicolumn{3}{|l|}{ Marital status } \\
\hline Unmarried & 75 & 65.2 \\
\hline Married & 23 & 20 \\
\hline Divorced & 17 & 14.8 \\
\hline Widowhood & 0 & 0 \\
\hline \multicolumn{3}{|l|}{ Educational level } \\
\hline Illiteracy and semi illiteracy & 1 & 0.9 \\
\hline Primary school & 6 & 5.2 \\
\hline Junior middle school & 15 & 13 \\
\hline $\begin{array}{l}\text { Senior high school/vocational high school/ } \\
\text { technical secondary school }\end{array}$ & 35 & 30.4 \\
\hline Junior college & 19 & 16.5 \\
\hline Bachelor degree or above & 39 & 33.9 \\
\hline \multicolumn{3}{|l|}{ Employment status } \\
\hline Employed & 92 & 80 \\
\hline Retired or & 6 & 5.2 \\
\hline Students at school & 3 & 2.6 \\
\hline Unemployed & 14 & 12.2 \\
\hline \multicolumn{3}{|l|}{ Personal monthly income (RMB) } \\
\hline$\leq 1000$ & 11 & 9.6 \\
\hline $1001-3000$ & 28 & 24.3 \\
\hline $3001-5000$ & 30 & 26.1 \\
\hline $5001-10000$ & 37 & 32.2 \\
\hline$>10000$ & 9 & 7.8 \\
\hline
\end{tabular}

\section{Behavioral characteristics}

Serological test results showed that 16 MSM were infected with HIV, and the other 99 MSM were negative. The HIV infection rate in this study was $13.9 \%(16 / 115)$.

Sexual partners: In the survey of male partners, having one sexual partner was the most common, the proportion of one male partner in the past month was $60.9 \%(n=70)$, while the proportion of one male partner in the past six months was $57.4 \%(\mathrm{n}=66)$. Among $101 \mathrm{MSM}$ with male partners in the past six months, the proportion of one male partner was $81.2 \%(n=82)$ which is the highest. In addition, the data showed that this group of MSM has the characteristics of multiple sexual partners (Table 2).

Sexual behaviour: Among 115 MSM, the percentage of having insertive sexual behaviors was $59.1 \%(n=68)$. All MSM who had insertive sexual behaviors had anal sexual behaviors, while the
Table 2: Characteristics of MSM sexual partners in Chongqing.

\begin{tabular}{|c|c|c|}
\hline Characteristics & $\mathbf{n}$ & $\%$ \\
\hline \multicolumn{3}{|l|}{ Male sexual partners } \\
\hline \multicolumn{3}{|c|}{ Number of male partners in the past month* } \\
\hline 0 & 32 & 27.8 \\
\hline 1 & 70 & 60.9 \\
\hline 2 & 9 & 7.8 \\
\hline$>2$ & 4 & 3.5 \\
\hline Total & 115 & 100 \\
\hline \multicolumn{3}{|c|}{ Number of male partners in the past 6 months* } \\
\hline 0 & 14 & 12.2 \\
\hline 1 & 66 & 57.4 \\
\hline 2 & 17 & 14.8 \\
\hline$>2$ & 18 & 15.6 \\
\hline Total & 115 & 100 \\
\hline \multicolumn{3}{|c|}{ Number of regular male partners in the past 6 months* } \\
\hline 0 & 12 & 11.8 \\
\hline 1 & 82 & 81.2 \\
\hline 2 & 5 & 5.0 \\
\hline$>2$ & 2 & 2.0 \\
\hline Total & 101 & 100 \\
\hline \multicolumn{3}{|c|}{ Number of casual male partners in the past 6 months* } \\
\hline 0 & 59 & 58.4 \\
\hline 1 & 24 & 23.8 \\
\hline 2 & 10 & 9.9 \\
\hline$>2$ & 8 & 7.9 \\
\hline Total & 101 & 100 \\
\hline \multicolumn{3}{|l|}{ Female sexual partners } \\
\hline \multicolumn{3}{|c|}{ Number of female sexual partners* } \\
\hline 0 & 89 & 77.4 \\
\hline 1 & 19 & 16.5 \\
\hline 2 & 6 & 5.2 \\
\hline$>2$ & 1 & 0.9 \\
\hline Total & 115 & 100 \\
\hline
\end{tabular}

*Indicates loss of data

proportion of having oral sexual behavior was $66.2 \%$ (45/68) (Table 3).

Condom use: In the survey of condom use when having sex with male partners, the percentage of condom use was $80.0 \%(n=92)$. Among 68 people who had anal sex in the past month, nine (13.2\%) MSM did not use condoms. Among 45 people who had oral sex in the past month, twenty-eight MSM did not use condoms (62.2\%).

Sixty-nine MSM reported condom use when having sex with female partners (both regular and casual), of which $47.8 \%(n=33)$ did not use condoms at all.

Among the subjects, thirty-seven MSM had regular female partners (including wives or girlfriends), seventeen (45.9\%) of whom did not use condoms frequently. Of the 27 people who reported condom use, six MSM (22.2\%) did not use condom all the way. In addition, in 37 
MSM with regular female partners, thirteen (35.1\%) of them did not use condoms in the whole process of their last sexual behavior (Table 4).

\section{Other behaviors}

In this study, the proportion of commercial sex and drug use in the past half year was low, accounting for $2.6 \%(\mathrm{n}=3)$ and $1.7 \%(\mathrm{n}=2)$, respectively. In the past six months $49.6 \%(n=57)$ of them searched for sexual partners through the Internet, and $5.2 \%(n=6)$ were diagnosed with sexually transmitted diseases (STDs) (Table 5).

\section{Discussion}

The HIV infection rate of MSM in this study was $13.9 \%$, higher than that of other key groups reported in some literature. For example, female sex workers: the global HIV infection rate of female sex workers was $10.4 \%$ in 2006-2017 [17], and $0.2 \%$ in China in 2018 [18]. Drug users: the HIV infection rate of drug users in five provinces of China was 10.9\% in 1995-2001 [19]; as of December 2013, the overall median HIV infection rate was $8 \%$ of 23 countries in the Middle East and North Africa inject drugs [20]; In 2018, the HIV infection rate of injecting drug users in China was 5.9\%, including 5.7\% for men and $7.2 \%$ for women [18]. Prisoners: the global HIV infection rate of prisoners was about 3.8\% [21]. Transgender people: the HIV infection rate of transgender women in Tianjin and Shanghai, China was $3.4 \%$ and $12.4 \%$ [22], Thailand was $13.5 \%$ [23], and Cambodia was $5.9 \%$ [24]. Sexual partners of key groups: $4 \%$ of male clients of female sex workers in Tijuana, Mexico, 2008 [25], and 10.2\% of male clients of female sex workers in Hanoi and Ho Chi Minh, Vietnam, 2013-2014 [26]. This result once again emphasizes that MSM is a high-risk group of HIV infection [7,8], so that the prevention and control of AIDS in this group should be paid attention [12].

Table 3: Sexual behavior.

\begin{tabular}{|l|c|c|}
\hline \multicolumn{1}{|c|}{ Characteristics } & \% \\
\hline Sexual behavior in the past month \\
\hline Number of insertive sexual behavior* \\
\hline 0 & 47 & 40.9 \\
\hline 1 & 31 & 26.9 \\
\hline 2 & 13 & 11.3 \\
\hline$>2$ & 24 & 20.9 \\
\hline Total & 115 & 100.0 \\
\hline Number of anal sexual behavior & & \\
\hline 0 & 0 & 0.0 \\
\hline 1 & 32 & 47.1 \\
\hline 2 & 12 & 17.6 \\
\hline$>2$ & 24 & 35.3 \\
\hline Total & 68 & 100.0 \\
\hline Number of oral sexual behavior* & 23 & 33.8 \\
\hline 0 & 21 & 30.9 \\
\hline 1 & 10 & 14.7 \\
\hline 2 & 14 & 20.6 \\
\hline$>2$ & 68 & 100.0 \\
\hline Total & \multicolumn{2}{|l|}{} \\
\hline
\end{tabular}

*Indicates loss of data.
Table 4: Condom use of MSM in Chongqing.

\begin{tabular}{|l|c|c|}
\hline Characteristics & $\mathbf{n}$ & $\%$ \\
\hline Condom use when having sex with a male partner* \\
\hline Yes & 92 & 80.0 \\
\hline No & 23 & 20.0 \\
\hline Total & 115 & 100.0 \\
\hline
\end{tabular}

Frequency of condom use in anal sex with a male partner in the past month

\begin{tabular}{|l|c|c|}
\hline 0 & 9 & 13.2 \\
\hline 1 & 34 & 50.0 \\
\hline 2 & 8 & 11.8 \\
\hline$>2$ & 17 & 25.0 \\
\hline Total & 68 & 100.0 \\
\hline
\end{tabular}

Frequency of condom use in oral sex with a male partner in the past month*

\begin{tabular}{|l|c|c|}
\hline 0 & 28 & 62.2 \\
\hline 1 & 13 & 28.9 \\
\hline 2 & 3 & 6.7 \\
\hline$>2$ & 1 & 2.2 \\
\hline Total & 45 & 100.0 \\
\hline
\end{tabular}

Frequency of condom use in anal sex with male partners in the past 6 months

\begin{tabular}{|l|c|c|}
\hline Always & 69 & 74.2 \\
\hline Occasionally & 17 & 18.3 \\
\hline Never & 7 & 7.5 \\
\hline Total & 93 & 100.0 \\
\hline Col
\end{tabular}

Condom use when having sex with a female partner (regular partner and casual partner)

\begin{tabular}{|l|c|c|}
\hline Yes & 36 & 52.2 \\
\hline No & 33 & 47.8 \\
\hline Total & 69 & 100.0 \\
\hline
\end{tabular}

Condom use when MSM have sex with a regular female partner (wife or girlfriend)

Frequency of condom use during sexual behavior in the past three months

\begin{tabular}{|l|c|c|}
\hline Use every time & 16 & 43.2 \\
\hline Frequently used & 4 & 10.8 \\
\hline Use and do not use the same number of times & 4 & 10.8 \\
\hline Occasional use & 3 & 8.1 \\
\hline Never use & 10 & 27.0 \\
\hline Total & 37 & 100.0 \\
\hline
\end{tabular}

Whether to use condoms in the whole process of sexual behavior in the past three months

\begin{tabular}{|l|c|c|}
\hline Yes & 21 & 77.8 \\
\hline No & 6 & 22.2 \\
\hline Total & 27 & 100.0 \\
\hline
\end{tabular}

Whether the condom was used in the whole process in the last sexual behavior*

\begin{tabular}{|l|c|c|}
\hline Yes & 24 & 64.9 \\
\hline No & 13 & 35.1 \\
\hline Total & 37 & 100.0 \\
\hline
\end{tabular}

Will you use condoms every time when you have sex intercourse in the next three months

\begin{tabular}{|l|l|l|}
\hline No & 4 & 10.8 \\
\hline Not sure & 7 & 18.9 \\
\hline
\end{tabular}

Citation: Ou Y, Pan H, Liu J, Lin B, Shi G, et al. (2020) AIDS-Related Behavioral Characteristics of Men Who Have Sex with Men in Chongqing, China. J Epidemiol Public Health Rev 5(2): dx.doi.org/10.16966/2471-8211.193 


\begin{tabular}{|l|c|c|}
\hline Yes & 26 & 70.3 \\
\hline Total & 37 & 100.0 \\
\hline Will condoms be used as much as possible in the next three months* \\
\hline No & 3 & 8.1 \\
\hline Not sure & 7 & 18.9 \\
\hline Yes & 27 & 73.0 \\
\hline Total & 37 & 100.0 \\
\hline
\end{tabular}

*Indicates loss of data

Table 5: Other AIDS- related behaviors of MSM in Chongqing.

\begin{tabular}{|l|c|c|}
\hline \multicolumn{1}{|c|}{ Characteristics } & n & $\%$ \\
\hline Whether a commercial sexual service in the past 6 & month* \\
\hline Yes & 3 & 2.6 \\
\hline No & 112 & 97.4 \\
\hline Total & 115 & 100.0 \\
\hline Whether illicit drugs used in the last half year \\
\hline Yes & 2 & 1.7 \\
\hline No & 113 & 98.3 \\
\hline Total & 115 & 100.0 \\
\hline
\end{tabular}

Frequency of Internet-seeking sexual partners in the last 6 months

\begin{tabular}{|l|c|c|}
\hline Often & 6 & 5.2 \\
\hline Sometimes & 14 & 12.2 \\
\hline Occasionally & 37 & 32.2 \\
\hline Never & 58 & 50.4 \\
\hline Total & 115 & 100.0 \\
\hline Diagnosed with STDs in the past six months & \multicolumn{2}{|l|}{} \\
\hline Yes & 6 & 5.2 \\
\hline No & 109 & 94.8 \\
\hline Total & 115 & 100.0 \\
\hline
\end{tabular}

*Indicates loss of data

\section{Social demographic factors related to HIV infection}

In this study, MSM is mainly young people, which is similar to the research results of many literatures [8,10,13,14,27-29]. Qin et al. [7] found that most HIV infection cases in China occurred in MSM aged 20-39, which indicates that HIV may be more prevalent among young MSM population in the sexually active period [13].

This study found that the majority of MSM were unmarried, which is consistent with the previous studies $[8,10,14,27,30]$. The first reason may be that same-sex marriage is not recognized by the law and the secular in China [28], while some studies have found that attitudes towards sex and gender diversity have become more tolerant in China, and more MSM will remain single or live with gay partners [29]. Second, some people hold the belief that not getting married is responsible for themselves and will not cause harm to female partners [31]. The third may be that unmarried people are freer, and their sexual behavior is not constrained by marriage and love relationships. In addition, the results also observed that $20.0 \%$ of MSM have been married, which is similar to the results of a cross-sectional survey from China [10]. Forced by social responsibility, and Chinese traditional culture (filial piety) or values (inheritance of family blood), the resulting family pressure (marriage and birth) and the protection of homosexuality from discrimination and stigmatization [10,11,14,28,29,31], some MSM may choose to get marriage with women $[28,29,32]$.

\section{Risk behaviors related to HIV infection}

The subjects in this study have the characteristics of multiple sexual partners, including multiple regular male partners, multiple casual male partners and multiple female partners. While multiple sexual partners were proved to be associated with HIV infection [12,14]. The characteristics of multiple sexual partners would lead to form a larger and more complex high-risk sexual network [14], so that MSM could have more opportunities to contact various sexual behaviors and potential partners with HIV positive [33]. Therefore, to promote the reduction of sexual partners to MSM [12] can be one of the important prevention and control measures.

In the study, the probability of anal sex between MSM and male partner was very high, which is consistent with the previous literature [14]. Meanwhile, when MSM have anal sex with male partners, the risk of HIV infection between them is the highest [27], but correct condom use can effectively prevent HIV infection [15,34,35]. The present study showed that $74.2 \%$ of MSM used condoms in every anal sex with their male partners in the past six months, which is higher than the results of a continuous cross-sectional survey conducted by Zhang and others in Chongqing from 2013 to 2017 [36]. It indicates that in recent years, MSM people have significantly increased their awareness of self-protection and safety sexual behaviors. However, 25.8\% of MSM (self-reported frequency of condom use during anal sex in the past 6 months) did not entirely use condom throughout their sex intercourse. Because these MSM prefer to have better sexual feelings and fear of distrust to their partners, they seldom use condoms in the process of sexual behavior [6]. And unprotected sex increases the risk of HIV infection in MSM and their male partners [10]. In addition, similar to other studies $[8,14]$, MSM has a high proportion of oral sex with male partners, which may be due to the fact that oral sex has proved to be a sexual behavior with a lower risk of HIV compared with anal sex $[15,37]$. However, the proportion of those who used condoms in oral sex was not high, and it cannot be ignored that HIV can be transmitted by oral sex [38]. Therefore, in order to reduce the risk of HIV infection in MSM and male partners when they have insertive sex (anal sex, oral sex), the importance of condoms use in the whole process and correctly during insertive sex behavior to MSM population should be emphasized, and adopting a safer way of sexual intercourse in this group need to be suggested.

In addition to the above high-risk sexual behavior, the MSM in this study also showed bisexual behavior. Similar to the previous results $[8,9,10,14]$, the proportion of condom use in the sexual behavior of MSM with female partners is not high: nearly half of MSM did not use condom when they had sex with female partners, and more than half of MSM with regular female partners reported that they did not use condom in each sexual behavior with their wives or girlfriends in the last three months. More than half of MSM with fixed female partners reported that they had not used condoms in every sexual act in the past three months. This study also found that MSM had more unprotected sexual behaviors with female partners than with men, probably because of family pressure or personal desire to have children of their own blood [14,29], or fear that the use of condoms will lead to distrust of their female partners, or lack of awareness of their own risks [14]. It is worth noting that MSM with bisexual behavior can become a bridge for transmitting HIV to the general female population $[8,9,11,39]$. Moreover, stigma and discrimination make MSM hidden themselves $[10,31,32]$. MSM rarely shows their true identity to their wife or their female partners, which makes the female partners may not know their male partner's sexual behavior and the coming risk of HIV infection [14]. Therefore, more attention should be paid to 
the population of MSM and their heterosexual partners, HIV testing and counseling (CHTC) should be provided to couples by pre-marital inspection, prenatal inspection and other links [40], and agree on targeted prevention, care and treatment programs based on husband and wife according to the test results, so as to reduce the possibility of HIV transmission to HIV negative partners.

Sex trade is considered an important factor in the risk of HIV infection [41]. At present, China is facing the challenge of spreading HIV from high-risk groups to the general population through homosexual activities [7,37], especially commercial sexual activities [37]. According to the existing data, the proportion of MSM engaged in commercial activities in China is not high (3.7\%-5.8\%) [29,32,34,42], which is consistent with the current research results (2.6\%). However, studies have shown that this group of MSM with commercial sexual behavior is more likely to be infected with HIV than those without commercial sexual behavior [42]. Thus, the follow-up research should continue to pay attention to this behavior of MSM, and advocate to them to minimize commercial sex, or to conduct protected safe sex in commercial sex activities.

It is reported that there are risk behaviors of drug use in MSM in China [6]. The drug use rate of MSM participating in this study was $1.7 \%$, far lower than that of MSM reported in Shenyang, China (23.2\%) [43] in 2014 and Beijing, China (27.5\%) in 2017 [44]. However, The current study results are similar to the results of a large-scale systematic analysis published in 2019 (1.5\% of MSM in China use drugs), which shows that, consistent with the findings of Dong MJ, et al. [10], drug use in Chongqing, China is not an important factor for MSM to infect HIV. It is worth noting that Zhang C, et al. [44] found that the HIV infection rate of MSM with drug abuse behavior was significantly higher than that of non drug users, suggesting it is still necessary to strengthen the prevention and control of drugs, strengthen the investigation and education of drug users, and pay attention to the publicity of drug use risk knowledge in AIDS risk education.

With the advantages of convenience, anonymity and security, and the ability to expand the social scope of MSM population (that is, to provide more choices to find friends and sexual partners), the Internet has become the most important way for MSM to find gay related information and sexual partners [14,27,32]. Compared with the current research results [30,45], the proportion of these subjects looking for sexual partners through the Internet is not high, which indicates that nearly half of these respondents may prefer to look for sexual partners in sauna, massage room and other places. And a cross-sectional study showed that MSM seeking partners in bathrooms, saunas and massage rooms had the highest HIV prevalence [10]. Therefore, the current study should suggest that the relevant departments strengthen the network supervision of dating software and dating platform, create a good and safe network environment as much as possible, increase the management and control of risk places such as sauna, massage room and men's bar, and effectively reduce similar risk places by compulsory means, so as to promote MSM to choose partners from a safer way.

As it is well known that STDs refers to diseases caused by pathogens acquired and transmitted through sexual activities [34], and sexual transmission is also one of the main ways of HIV infection $[11,13,46]$. Studies have shown that both ulcerative diseases (such as genital herpes, syphilis and chancre) and non ulcerative diseases were associated with an increased risk of HIV infection, susceptibility and transmission $[34,47]$. Because of their similar transmission routes, STDs can be used as a synergistic factor of HIV transmission, and infection with
STDs can increase the risk of HIV infection. In this study, $5.2 \%$ of MSM self-reported had been diagnosed with STDs, which was lower than the prevalence of sexually transmitted diseases (syphilis) in 2011 in Harbin (16.4\%) [42], 2013-2014 in Hangzhou (8.5\%) [8] and 20132014 in Wenzhou (9.7\%) [14]. The self-reported STDs prevalence of this study was not high, but there may be no reported or no STDs screening. Therefore, attention should be paid to STDs screening work of MSM population. AIDS related consultation and effectively intervention and treatment should be provided to MSM with other STDs diagnosis.

\section{Conclusions}

There are multiple sexual partners, unprotected sexual behaviors, bisexual sexual behaviors and other risky behaviors related to HIV transmission infection in the MSM population in Chongqing. These behaviors have jointly established a complex sexual network, which will help HIV spread in Chongqing.

There are multiple sexual partners, unprotected sexual behaviors, bisexual behaviors and other risk behaviors related to HIV transmission and infection in MSM population in Chongqing, China. These behaviors have jointly established a complex sexual network, which will contribute to the prevalence of HIV in Chongqing. Thus, in order to prevent HIV transmission between MSM and their partners, targeted prevention and control measures should be formulated based on the social demographic and behavioral characteristics.

\section{Competing Interests}

The authors declare no conflict of interest.

\section{Author Contributions}

Data curation, Yanran Ou and Hong Pan; Formal analysis, Yanran $\mathrm{Ou}$; Investigation, Hong Pan, Jiaxiu Liu, Bing Lin and Guiqian Shi; Methodology, Yanran Ou and Xiaoni Zhong; Project administration, Xiaoni Zhong; Software, Yanran Ou, Jiaxiu Liu and Bing Lin; Supervision, Xiaoni Zhong; Writing-original draft, Yanran Ou; Writing-review \& editing, Xiaoni Zhong.

\section{Acknowledgments}

We thank all participants and investigators in Chongqing, China.

\section{Funding}

This work was supported by the National Key Project for Infectious Diseases of the Ministry of Science and Technology of China (2018ZX10721102-005).

\section{References}

1. World Health Organization (1984) Acquired Immunodeficiency Syndrome--An Assessment of the Present Situation in the World: Memorandum from a WHO Meeting. Bull World Health Organ 62: 419-432.

2. Alfvén T, Erkkola T, Ghys PD, Padayachy J, Warner-Smith M, et al. (2017) Global AIDS Reporting-2001 to 2015: Lessons for Monitoring the Sustainable Development Goals. AIDS Behav 21: 5-14.

3. Joint United Nations Programme on HIV/AIDS (2020) Global HIV \& AIDS statistics-2019 fact sheet. Geneva, Switzerland.

4. Joint United Nations Programme on HIV/AIDS (2019) UNAIDS Data 2019. Geneva, Switzerland.

5. Joint United Nations Programme on HIV/AIDS (2020) Country factsheets CHINA 2018. Geneva, Switzerland. 
6. Chow EPF, Lau JTF, Zhuang X, Zhang X, Wang Y, et al. (2014) HIV Prevalence Trends, Risky Behaviours, and Governmental and Community Responses to the Epidemic Among Men Who Have Sex With Men in China. Biomed Res Int 2014: 607261.

7. Qin Q, Guo W, Tang W, Mahapatra T, Wang L, et al. (2017) Spatial Analysis of the Human Immunodeficiency Virus Epidemic Among Men Who Have Sex With Men in China, 2006-2015. Clin Infect Dis 64: 956-963.

8. Li R, Wang H, Pan X, Ma Q, Chen L, et al. (2017) Prevalence of Condomless Anal Intercourse and Recent HIV Testing and Their Associated Factors Among Men Who Have Sex With Men in Hangzhou, China: A Respondent-Driven Sampling Survey. PLoS One 12: e0167730.

9. Chow EPF, Wilson DP, Zhang J, Jing J, Zhang L (2011) Human Immunodeficiency Virus Prevalence is Increasing Among Men Who Have Sex With Men in China: Findings From a Review and MetaAnalysis. Sex Transm Dis 38: 845-857.

10. Dong MJ, Peng B, Liu ZF, Ye QN, Liu H, et al. (2019) The Prevalence of HIV Among MSM in China: A Large-Scale Systematic Analysis. BMC Infect Dis 19: 1000.

11. Yan Y, Wu S, Chen L, Yan P, Qiu Y, et al. (2016) Shift in HIV/AIDS Epidemic in Southeastern China: A Longitudinal Study From 1987 to 2015. Int J Environ Res Public Health 13: 794

12. Wang N, Wu G, Lu R, Feng L, Xiao Y, et al. (2016) Investigating HIV Infection and HIV Incidence Among Chinese Men Who Have Sex With Men With Recent Sexual Debut, Chongqing, China, 2011. AIDS Behav 20: 2976-2982.

13. Yang S, Chiu APY, Lin Q, Zeng Z, Li Y, et al. (2018) HIV Epidemics in Shenzhen and Chongqing, China. PLoS One 13: e0192849.

14. Ma Q, Zeng S, Xia S, Pan X, Wang D, et al. (2015) Risky Sexual Networks and Concentrated HIV Epidemics Among Men Who Have Sex With Men in Wenzhou, China: A Respondent-Driven Sampling Study. BMC Public Health 15: 1246.

15. Joint United Nations Programme on HIV/AIDS (2020) HIV and AIDSBasic facts. Geneva, Switzerland.

16. Valerio MA, Rodriguez N, Winkler $\mathrm{P}$, Lopez J, Dennison $\mathrm{M}$, et al (2016) Comparing Two Sampling Methods to Engage Hard-To-Reach Communities in Research Priority Setting. BMC Med Res Methodol 16: 146.

17. Shannon K, Crago AL, Baral SD, Bekker LG, Kerrigan D, et al. (2018) The Global Response and Unmet Actions for HIV and Sex Workers. Lancet 392: 698-710.

18. Joint United Nations Programme on HIV/AIDS (2020) The Key Populations Atlas. Geneva, Switzerland.

19. Wang L, Guo W, Li D, Ding Z, McGoogan JM, et al. (2015) HIV Epidemic Among Drug Users in China: 1995-2011. Addiction 110: 20-28.

20. Mumtaz GR, Weiss HA, Thomas SL, Riome S, Setayesh $\mathrm{H}$, et al. (2014) HIV Among People Who Inject Drugs in the Middle East and North Africa: Systematic Review and Data Synthesis. PLoS Med 11: e1001663.

21. Dolan K, Wirtz AL, Moazen B, Ndeffo-Mbah M, Galvani A, et al. (2016) Global Burden of HIV, Viral Hepatitis, and Tuberculosis in Prisoners and Detainees. Lancet 388: 1089-1102.

22. Shan D, Yu MH, Yang J, Zhuang MH, Ning Z, et al. (2018) Correlates of HIV Infection Among Transgender Women in Two Chinese Cities. Infect Dis Poverty 7: 123.
23. Guadamuz TE, Wimonsate $W$, Varangrat $A$, Phanuphak $P$, Jommaroeng R, et al. (2011) HIV Prevalence, Risk Behavior, Hormone Use and Surgical History Among Transgender Persons in Thailand. AIDS Behav 15: 650-658.

24. Chhim S, Ngin C, Chhoun P, Tuot S, Ly C, et al. (2017) HIV Prevalence and Factors Associated With HIV Infection among Transgender Women in Cambodia: Results From a National Integrated Biological and Behavioral Survey. BMJ Open 7: e015390.

25. Patterson TL, Goldenberg S, Gallardo M, Lozada R, Semple SJ, et al. (2009) Correlates of HIV, Sexually Transmitted Infections, and Associated High-Risk Behaviors Among Male Clients of Female Sex Workers in Tijuana, Mexico. AIDS 23: 1765-1771.

26. Nadol P, Hoang TV, Le LV, Nguyen TA, Kaldor J, et al. (2017) High HIV Prevalence and Risk Among Male Clients of Female Sex Workers in Hanoi and Ho Chi Minh City, Vietnam. AIDS Behav 21: 2381-2393.

27. Ruan Y, Wu G, Lu H, Xiao Y, Zhao Y, et al. (2014) Sexual partnerships with men and women among men who have sex with men in Beijing and Chongqing, China, 2010. AIDS Behav 18: 180-188.

28. Yang Z, Li S, Zhang R, Na J, Li Y, et al. (2020) Current Heterosexual Marriage Is Associated With Significantly Decreased Levels of Anxiety Symptoms Among Chinese Men Who Have Sex With Men. BMC Psychiatry 20: 151.

29. Wu W, Yan X, Zhang X, Goldsamt L, Chi Y, et al. (2020) Potential HIV Transmission Risk among Spouses: Marriage Intention and Expected Extramarital Male-To-Male Sex Among Single Men Who Have Sex With Men in Hunan, China. Sex Transm Infect 96: 151-156.

30. He J, Xu HF, Cheng WB, Zhang SJ, Gu J, et al. (2018) Intimate Relationship Characteristics as Determinants of HIV Risk Among Men Who Have Sex With Regular Male Sex Partners: A CrossSectional Study in Guangzhou, China. BMC Infect Dis 18: 150.

31. Steward WT, Miège P, Choi KH (2013) Charting a Moral Life: The Influence of Stigma and Filial Duties on Marital Decisions Among Chinese Men Who Have Sex With Men. PLoS One 8: e71778.

32. Feng Y, Wu Z, Detels R (2010) Evolution of Men Who Have Sex With Men Community and Experienced Stigma Among Men Who Have Sex With Men in Chengdu, China. J Acquir Immune Defic Syndr 53: S98-S103.

33. Beyrer C, Baral SD, van Griensven F, Goodreau SM, Chariyalertsak S, et al. (2012) Global Epidemiology of HIV Infection in Men Who Have Sex With Men. Lancet 380: 367-377.

34. Workowski KA, Bolan GA (2015) Sexually Transmitted Diseases Treatment Guidelines, 2015. MMWR Recomm Rep 64: 1-137.

35. Tao L, Liu M, Li S, Liu J, Wang N (2018) Condom Use in Combination With ART Can Reduce HIV Incidence and Mortality of PLWHA Among MSM: A Study From Beijing, China. BMC Infect Dis 18: 124.

36. Zhang Y, Wu G, Lu R, Xia W, Hu L, et al. (2019) What Has Changed HIV and Syphilis Infection among Men Who Have Sex With Men (MSM) in Southwest China: A Comparison of Prevalence and Behavioural Characteristics (2013-2017). BMC Public Health 19: 1314

37. Glynn TR, Operario D, Montgomery M, Almonte A, Chan PA (2017) The Duality of Oral Sex for Men Who Have Sex With Men: An Examination Into the Increase of Sexually Transmitted Infections Amid the Age of HIV Prevention. AIDS Patient Care STDS 31: 261267.

38. Gilbart VL, Evans BG, Dougan S (2004) HIV Transmission Among Men Who Have Sex With Men Through Oral Sex. Sex Transm Infect 80: 324 
39. Choi KH, Gibson DR, Han L, Guo Y (2004) High Levels of Unprotected Sex With Men and Women Among Men Who Have Sex With Men: A Potential Bridge of HIV Transmission in Beijing, China. AIDS Educ Prev 16: 19-30.

40. World Health Organization. (2012) Guidance on Couples HIV Testing and Counselling Including Antiretroviral Therapy for Treatment and Prevention in Serodiscordant Couples: Recommendations for a Public Health Approach. Geneva, Switzerland.

41. Ranganathan M, Heise L, Pettifor A, Silverwood RJ, Selin A, et al. (2016) Transactional Sex Among Young Women in Rural South Africa: Prevalence, Mediators and Association With HIV Infection. J Int AIDS Soc 19: 20749.

42. Zhang L, Zhang D, Yu B, Wang S, Liu Y, et al. (2013) Prevalence of HIV Infection and Associated Risk Factors Among Men Who Have Sex With Men (MSM) in Harbin, P. R. China. PLoS One 8: e58440.

43. Xu JJ, Qian HZ, Chu ZX, Zhang J, Hu QH, et al. (2014) Recreational Drug Use Among Chinese Men Who Have Sex With Men: A Risky Combination With Unprotected Sex for Acquiring HIV Infection. Biomed Res Int 2014: 725361.
44. Zhang C, Qian HZ, Yin L, Liu Y, Strauss SM, et al. (2016) Sexual Behaviors Linked to Drug and Alcohol Use Among Men Who Have Sex With Men in China. Subst Use Misuse 51: 1821-1830.

45. Liu J, Zhong X, Lu Z, Peng B, Zhang Y, et al. (2020) Anxiety and Depression Associated With Anal Sexual Practices Among HIVNegative Men Who Have Sex With Men in Western China. Int J Environ Res Public Health 17:464.

46. Petroll $A E$, Hare CB, Pinkerton SD (2008) The Essentials of HIV: A Review for Nurses. J Infus Nurs 31: 228-235.

47. Fleming DT, Wasserheit JN (1999) From Epidemiological Synergy to Public Health Policy and Practice: The Contribution of Other Sexually Transmitted Diseases to Sexual Transmission of HIV Infection. Sex Transm Infect 75: 3-17. 